\title{
PHYSIOLOGICAL REACTION TO WORK IN COLD MICROCLIMATE
}

\section{ALICJA BORTKIEWICZ ${ }^{1}$, ELŻBIETA GADZICKA ${ }^{1}$, WIESŁAW SZYMCZAK ${ }^{2}$, AGATA SZYJKOWSKA ${ }^{1}$, WIESŁAWA KOSZADA-WŁODARCZYK ${ }^{1}$, and TERESA MAKOWIEC-DĄBROWSKA ${ }^{1}$}

${ }^{1}$ Department of Work Physiology and Ergonomics

${ }^{2}$ Deparment of Environmental Epidemiology

Nofer Institute of Occupational Medicine

Lódź, Poland

\begin{abstract}
Objectives: In Poland, occupational exposure to cold microclimate is quite common (5.1 workers/1000 occupationally active people). Reports on health effects of this exposure are rather scarce. The aim of the study was to evaluate the physiological reaction in workers occupationally exposed to cold microclimate. Materials and Methods: Examinations were performed in a group of 102 workers ( 41 women and $61 \mathrm{men}$ ) employed at cold storage units. The mean age in the group was $39.1 \pm 9.9$ years and the duration of employment under conditions of cold environment was over 12 years. The study population was divided into four groups, according to microclimate conditions (group I, ambient temperature $-26^{\circ} \mathrm{C}$; group II, $10-14^{\circ} \mathrm{C}$; group III, $18-20^{\circ} \mathrm{C}$, control group; and group IV, $0-10^{\circ} \mathrm{C}$ ). The workers underwent the following procedures: general medical examinations, cold pressor test, ambulatory blood pressure monitoring, and heart rate variability (HRV) analysis (time- and frequency-domain parameters). Results: The results were adjusted for confounding factors (age, smoking and drinking habits). The analysis of HRV parameters did not reveal any significant differences between the study groups. However, systolic and diastolic blood pressure (BP) in the daytime and at night was significantly higher in group IV compared to group II. Mean heart rate (HR) in the daytime and at night and the BP and HR day/night ratio did not differ between the groups. The analysis of BP by gender revealed that in women, systolic BP during the day and at night was significantly higher in group IV than in group II. In the group of workers with hypertension (18 men and 5 women), men reacted to the cold pressor test either by increased or decreased BP while all the women reacted by the increased BP. Conclusions: Our findings indicated that in workers exposed to cold microclimate, the physiological reaction was dependent on gender and ambient temperature. Women seemed to be more sensitive to cold stress than men. However, this finding must be further investigated.
\end{abstract}

Key words:

Ambulatory blood pressure monitoring, Heart rate variability, Cold pressor test, Occupational exposure to cold

\section{INTRODUCTION}

Cold environment is one of the factors that may induce adverse health effects. However, this problem remains largely unsolved [1]. Most of the studies intended to explain the control mechanisms of systemic functions under conditions of exposure to cold microclimate have been performed in laboratories and involved intensive but rather shortterm exposures [2,3]. Reports on physiological reactions and health effects of long-term occupational exposure to moderately low temperatures are rather scarce. In Poland, occupational exposure to cold microclimate is quite common; it concerns 30874 workers (5.1 workers per 1000 occupationally active people). Exposure to low temperatures is a major problem for people working in the open air (e.g., forestry, agriculture, municipal services or transportation workers) and those employed at some branches of the food industry (frozen fruit, vegetables, meat, ice cream or other dairy products). Among forestry workers, exposure to cold microclimate applies to 31.9 per 1000 employees, while the corresponding ratio for the food industry work-

This study was performed as a part of the project "Professional Activity of Workers and the Ageing Society" (PCZ 21-21/6).

Received: February 8, 2006. Accepted: June 12, 2006.

Address reprint requests to Assoc. Prof. A. Bortkiewicz PhD, Nofer Institute of Occupational Medicine, św. Teresy 8, 91-348 Łódź, Poland (e-mail: ala@sunlib.p.lodz.pl). 
ers is $29.3 / 1000$. The number of workers exposed to low temperature continues to grow. Therefore, it seems desirable to explain the physiological reaction to work in cold microclimate, which will help improve workers' protection against adverse health effects.

It is likely that a long-term exposure to cold environment may significantly affect the activity of the autonomic nervous system, which can lead to cardiovascular disturbances (e.g., arterial hypertension) [4]. The role of the neurovegetative regulation is to adjust the cardiac function and vascular response to different activities and states of the cardiovascular system (sleep, mental and physical effort, various emotional states) under varied environmental conditions, such as ambient temperature, atmospheric pressure, etc. The regulation is effected through the autonomic nervous system and the hormones produced by neurosecretory neurons [5]. The aim of our study was to assess the physiological reactions (arterial blood pressure, heart rate (HR), heart rate variability (HRV)) in workers occupationally exposed to cold microclimate. The HRV analysis was performed since it helps assess the sympathetic and parasympathetic activity of the autonomic nervous system and thermoregulatory processes $[5,6]$. The method has become commonly used in the diagnosis of diabetic neuropathy and risk assessment of sudden cardiac death in post-infarction patients as well as in screening other cardiovascular and neurological disorders [6-11]. It is also used in experimental physiological studies [12,13], but rarely in examinations of workers exposed to various occupational hazards [14].

Ambulatory blood pressure monitoring (ABPM) makes it possible to identify people with high risk of developing arterial hypertension [15-17]. This method is also effective in monitoring arterial hypertension, particularly during the unstable period of the disease as it eliminates the "white coat hypertension" effect [18-20]. People under ABPM are able to carry out their normal professional and daily activities.

\section{MATERIALS AND METHODS}

The study was approved by the Regional Biomedical Ethics Committee. All subjects were informed about the study protocol and submitted their written consent.
We have decided to examine people at varied levels of exposure to different ambient temperatures. Examinations were performed in a group of 102 (41 women and 61 men) cold storage workers, aged $39.1 \pm 9.9$ years with more than 12 years of employment under conditions of cold microclimate. The men and women did not differ with respect to age and overall employment duration. However, the women had a significantly longer history of exposure to cold microclimate at work (Table 1). The study population was divided into four groups, each employed under different microclimate conditions (Table 2):

$\square$ Group I - 28 freeze room workers, exposure to ambient temperature $-26^{\circ} \mathrm{C}$ for about $30 \mathrm{~min}$ per day. The male workers were involved in pork-half loading and unloading. Energy expenditure for this job was calculated at 1660-2020 kcal per work shift. This range corresponded with heavy or very heavy physical work, with high static workload and medium work strain. The female workers performed light auxiliary work with low static workload and low level of monotype movements.

- Group II - 44 workers involved in foodstuff (icecream and noodle) production. Their job involved exposure to ambient temperature between $10^{\circ} \mathrm{C}$ and $14^{\circ} \mathrm{C}$ for

Table 1. Characteristics of study population

\begin{tabular}{lccc}
\hline \multicolumn{1}{c}{ Variables } & Total & Females & Males \\
\hline No. of workers & 102 & 41 & 61 \\
Age & $39.1 \pm 9.9$ & $41.1 \pm 8.2$ & $38.8 \pm 10.9$ \\
Employment duration & $20.6 \pm 10.3$ & $21.7 \pm 9.1$ & $19.9 \pm 11.0$ \\
$\begin{array}{l}\text { Period of work in cold } \\
\text { environment }\end{array}$ & $12.4 \pm 9.4$ & $16.2 \pm 9.7^{*}$ & $9.9 \pm 11.0$ \\
$\begin{array}{l}\text { Clo (thermal insulation of } \\
\text { clothes index) }\end{array}$ & $1.30 \pm 0.20$ & $1.35 \pm 0.26$ & $1.27 \pm 0.15$ \\
$\begin{array}{l}\text { Energy expenditure } \\
\text { (kcal/workshift) }\end{array}$ & - & $759 \pm 281$ & $1363 \pm 583^{*}$ \\
$\begin{array}{l}\text { Body mass index (BMI) } \\
\text { No. of smokers }\end{array}$ & $26.0 \pm 3.6$ & $24.3 \pm 3.2$ & $27.2 \pm 3.4$ \\
$\begin{array}{l}>10 \text { cigarettes a day) }(\%) \\
\begin{array}{l}\text { Diseases diagnosed } \\
\text { - Hypertension } \\
\text { - Diabetes mellitus type II }\end{array}\end{array}$ & $46(45)$ & $14(34)$ & $32(53)$ \\
- Subjective cardiovascular & $41(40)$ & $22(63)$ & $19(29)$ \\
$\quad$ symptoms (\%) & & & \\
\hline
\end{tabular}

BMI - body mass index = body mass $/$ height ${ }^{2}\left(\mathrm{~kg} / \mathrm{m}^{2}\right)$;

* Statistically significant difference $(\mathrm{p}<0.05)$. 
Table 2. Characteristics of the groups divided according to working conditions

\begin{tabular}{|c|c|c|c|c|}
\hline Variables & I & II & $\begin{array}{c}\text { III } \\
\text { Control group }\end{array}$ & IV \\
\hline $\begin{array}{l}\text { No. of workers } \\
\text { females (F)/males (M) }\end{array}$ & $\begin{array}{c}28 \\
4 / 24\end{array}$ & $\begin{array}{c}44 \\
26 / 18\end{array}$ & $\begin{array}{c}8 \\
2 / 6\end{array}$ & $\begin{array}{c}22 \\
7 / 15\end{array}$ \\
\hline Age & $40.5 \pm 8.7$ & $41.2 \pm 9.7$ & $45.4 \pm 8.5$ & $33.8 \pm 10.4^{*}$ \\
\hline Employment duration & $20.9 \pm 8.8$ & $22.3 \pm 10.5$ & $25.5 \pm 8.2$ & $15.3 \pm 10.8^{* *}$ \\
\hline Period of work in cold environment & $10.0 \pm 7.8$ & $15.5 \pm 10.2$ & $15.1 \pm 6.1$ & $8.5 \pm 8.4^{* * *}$ \\
\hline $\begin{array}{l}\text { Energy expenditure } \\
\text { kcal/workshift }\end{array}$ & $\begin{array}{l}\mathrm{F} \text { - light work } \\
\mathrm{M}-1660-2020\end{array}$ & $\begin{array}{r}750-1200 \\
1200-1700\end{array}$ & $\begin{array}{c}800-1120 \\
1150-1620\end{array}$ & $\begin{array}{l}1020-1190 \\
1130-1870\end{array}$ \\
\hline
\end{tabular}

Statistically significant difference $(\mathrm{p}<0.05)$ between groups:

* I-IV, II-IV, III-IV; $\quad * *$ II-IV, III-IV; $\quad * * *$ I-II, II-IV.

$8 \mathrm{~h} /$ day. Energy expenditure for female workers ranged from 750-1200 kcal, corresponding with medium-heavy, heavy or very heavy physical work, whereas for male workers it amounted to $1200-1700 \mathrm{kcal}$, which stood for medium-heavy or heavy physical work. Static workload in this group was assessed as medium or high. In most cases the work strain was also high.

- Group III - 8 workers employed in in-plant workshops under exposure to ambient temperature of $18-20^{\circ} \mathrm{C}$ for $8 \mathrm{~h} /$ day and performed medium-heavy or heavy physical work (1150-1620 kcal for male workers and 800-1120 kcal for female workers). The static workload was medium or high and the work strain was low or medium. This group did not meet the criteria for working in cold microclimate since the ambient temperature below $0^{\circ} \mathrm{C}$ occurred only occasionally, in emergency situations. Therefore, this group was assumed to be the control group.

$\square$ Group IV - 44 workers of the meat department at a hypermarket, with exposure ( $8 \mathrm{~h} /$ day $)$ to ambient temperature varying from $0^{\circ} \mathrm{C}$ to $10^{\circ} \mathrm{C}$. The male workers were engaged in cutting up pork-halves and handling activities. Their energy expenditure varied from 1130 to $1870 \mathrm{kcal}$, depending on the type of work performed. This range was classified as medium-heavy and heavy physical work. The female workers handled meat packaging that was assessed as heavy physical work (energy expenditure 1020-1190 kcal).

All the workers employed at workposts under study wore protective clothing as specified in occupational safety and health regulations.
All the examinations were performed in the winter season, during morning hours, in workers taking up their duties after the night rest. The workers underwent the following procedures:

$\square$ general medical examinations with office blood pressure (BP) measurements (systolic BPS_O and diastolic BPD_O);

$\square$ an interview oriented towards the risk and presence of ischemic heart disease and other cardiovascular diseases, including family history of metabolic and cardiovascular diseases;

m cold pressor test (CPT). It was performed on each subject after a 5-min rest. Before the test, systolic and diastolic BP (BPS_B, BPD_B) and heart rate (HR_B) were measured, using an Omron M5-I device. Then the subject immersed one hand in ice water $\left(3^{\circ} \mathrm{C}\right)$ for one min. Immediately after the test, blood pressure (BPS_A, BPD_A) and heart rate (HR_A) were checked again; ambulatory blood pressure monitoring (ABPM). It was performed during workers' regular occupational and other daily activities, using the Medilog ABPM System, and had to be properly coordinated with the working cycle. The measurements were carried out automatically, every half an hour during daily activities and every hour during sleep. Altogether, approximately 40 measurements were made for each worker. Mean systolic and diastolic blood pressure (BPS and BPD) and HR daytime activity (D) and night-time rest (N) were calculated with Staessen's standards of BP as the reference values [21]. The day-night ratios were determined for systolic and 
diastolic blood pressure (BPSD/BPSN, BPDD/BPDN) and for heart rate (HRD/HRN). The normal values of $\mathrm{BP}$ and $\mathrm{HR}$ ratios are 1.1 or more. Subjects with $\mathrm{BP}$ ratio lower than 1.1 are called non-deepers (subjects without the physiological decrease in blood pressure at night); $\square$ resting ECG with HRV analysis. HRV was analyzed using the Medea-HRV computer system (Gliwice, Poland), which consists of a three-channel low-noise biological amplifier, a 16-bit AD digitizer and IBM PC 486/33 MHz. The ECG signal was sampled at a rate of 2000 samples per second and filtered to remove the ectopic activity and artefacts prior to R-R interval file generation. The system is characterized by high accuracy of QRS detection; 512 consecutive, normal cardiac cycles were registered under resting conditions. Short-term ECG records were used to calculate non-spectral (time-domain) and spectral (frequency-domain) HRV indices. Statistical analysis made it possible to calculate the following parameters: mean R-R interval (AVG R-R); R-R standard deviation (STD R-R); median (MED), modal (MOD), minimum (MIN) and maximum (MAX) R-R values. R-R interval was the subject to Fast Fourier Transformation (FFT) using Blackman-Harris window. The power spectrum density (area covered by the power spectrum) was computed for the following frequency bands: very low (VLF): 0.0167-0.05 Hz; low (LF): $0.05-0.15 \mathrm{~Hz}$; high (HF): 0.15-0.35 Hz, ultra high (UHF): $0.35-0.50 \mathrm{~Hz}$, and expressed as a percentage of spectrum power in the $0.0-2.5 \mathrm{~Hz}$ range. According to the commonly adopted interpretation, the HF power spectrum is parasympathetic-mediated, while the LF one is sympathetic-mediated. The $\mathrm{LF} / \mathrm{HF}$ ratio indicates a sympathovagal balance $[6,12]$.

$\square$ For each type of workpost, the overall level of workload was determined based on estimates for energy expenditure (using the method of indirect calorimetry), static workload, and work strain due to monotype working movements according to the method of Kirschner and Kock.

$\square$ For each worker, the required clothing insulation quotient (IREQ) was determined. This indicator is calculated based on direct measurements of ambient temperature, air flow rate, mean heat radiation and energy expenditure. Based on the workers' reports on the kind of working clothes worn by them, the thermal insulation of clothes was calculated (clo).

\section{Statistical analysis}

The obtained results were analyzed with use of Student's t-test (for normal distributions) or the non-parametric Mann-Whitney test (for other distributions), the Chisquare test and analysis of variance with multiple comparison tests, Fisher's exact probability test, and multiple linear regression.

\section{RESULTS}

Medical examinations and interviews indicated that the study groups were similar with respect to the level of leisure time physical activity and the dietary habits. They differed with respect to age (Table 2). No significant differences could be found between the study groups regarding overall workload, static workload and work strain due to monotype working movements.

It was found that for the energy expenditure at the workposts under study, the required thermal insulation of clothes ranged from 0.6 to 1.30. Insulation of clothes worn by workers fell within the required range for this parameter [Table 1].

The statistical analysis showed that group IV was significantly $(\mathrm{p}<0.05)$ younger (mean age, $33.8 \pm 10.4$ years). Arterial hypertension was reported in the interview by 11 workers (4 women and 7 men). Our analysis of BP and HR on office examination revealed that BPD_O was significantly higher in groups I and III than in group IV (Table 3).

Mean systolic and diastolic blood pressure and HR values in ABPM measurement in all the study groups were normal [21]. The between-group comparison of results for ABPM revealed that mean $\mathrm{BP}$ in the daytime and at night was significantly higher in group IV than in group II. The mean HR, both in the daytime and at night, did not differ between the groups. The BP and HR day/night ratios did not show statistically significant differences (Table 3 ). In ABPM, six (21.4\%) workers from group I, three (6.8\%) from group II, one (12.5\%) from group III, and six (27.3\%) 
Table 3. Blood pressure and heart rate in the study groups

\begin{tabular}{|c|c|c|c|c|c|}
\hline $\begin{array}{c}\mathrm{BP} \\
(\mathrm{mm} \mathrm{Hg})\end{array}$ & I & II & III & IV & $\mathrm{P}$ \\
\hline BPS_O & $140.5 \pm 21.8$ & $134.2 \pm 16.6$ & $140.8 \pm 21.6$ & $129.0 \pm 12.6$ & NS \\
\hline BPD_O & $88.2 \pm 15.0$ & $84.2 \pm 11.3$ & $89.2 \pm 8.8$ & $77.5 \pm 5.2$ & $\begin{array}{c}0.0141 \\
\text { I-IV, } \\
\text { III-IV }\end{array}$ \\
\hline HR_O & $73.2 \pm 9.3$ & $72.5 \pm 9.3$ & $77.2 \pm 8.6$ & $71.8 \pm 10.0$ & NS \\
\hline BPSD & $134.3 \pm 19.1$ & $124.9 \pm 13.6$ & $132.8 \pm 8.2$ & $136.9 \pm 16.0$ & $\begin{array}{c}0.02 \\
\text { II-IV }\end{array}$ \\
\hline BPDD & $81.8 \pm 11.2$ & $77.8 \pm 8.8$ & $77.6 \pm 8.5$ & $79.8 \pm 10.3$ & NS \\
\hline HRD & $84.1 \pm 8.2$ & $82.4 \pm 10.8$ & $82.8 \pm 8.6$ & $85.8 \pm 10.4$ & NS \\
\hline BPSN & $120.0 \pm 23.8$ & $112.0 \pm 20.7$ & $122.1 \pm 15.6$ & $127.8 \pm 208$ & $\begin{array}{c}0.05 \\
\text { II-IV }\end{array}$ \\
\hline BPDN & $67.8 \pm 9.2$ & $67.4 \pm 10.6$ & $67.8 \pm 11.6$ & $67.6 \pm 13.8$ & NS \\
\hline HRN & $68.7 \pm 7.2$ & $69.4 \pm 10.2$ & $66.8 \pm 8.0$ & $69.2 \pm 10.0$ & NS \\
\hline BPSD//BPSN & $1.12 \pm 0.1$ & $1.13 \pm 0.1$ & $1.10 \pm 0.1$ & $1.09 \pm 0.1$ & NS \\
\hline BPDD/BPDN & $1.20 \pm 0.2$ & $1.17 \pm 0.1$ & $1.16 \pm 0.2$ & $1.22 \pm 0.2$ & NS \\
\hline HRD/HRN & $1.22 \pm 0.1$ & $1.21 \pm 0.2$ & $1.24 \pm 0.1$ & $1.26 \pm 0.2$ & NS \\
\hline
\end{tabular}

\begin{tabular}{cc}
\hline BP - blood pressure; & BPS_O - systolic blood pressure (office measurement); \\
BPD_O - diastolic blood pressure (office measurement); & HR_O - heart rate (office measurement); \\
BPSD - systolic blood pressure during the day; & BPDD - diastolic blood pressure during the day; \\
HRD - heart rate during the day; & BPSN - systolic blood pressure during the night; \\
BPDN - diastolic blood pressure during the night; & HRN - heart rate during the night; \\
BPSD/BPSN - night drop of systolic blood pressure; & BPDD/BPDN - night drop of diastolic blood pressure; \\
HRD/HRN - night drop of heart rate; & NS - not significant.
\end{tabular}

Table 4. Blood pressure and heart rate in workers relative to their working conditions

\begin{tabular}{|c|c|c|c|c|c|}
\hline & \multicolumn{5}{|c|}{ Females } \\
\hline $\begin{array}{c}\mathrm{BP} \\
(\mathrm{mg} \mathrm{Hg})\end{array}$ & $\begin{array}{c}\mathrm{I} \\
\mathrm{n}=4\end{array}$ & $\begin{array}{c}\text { II } \\
\mathrm{n}=26\end{array}$ & $\begin{array}{c}\text { III } \\
n=2\end{array}$ & $\begin{array}{c}\text { IV } \\
n=7\end{array}$ & $\mathrm{P}$ \\
\hline BPSD & $119.8 \pm 15.2$ & $119.4 \pm 11.0$ & $127.0 \pm 8.2$ & $130.7 \pm 15.9$ & $\begin{array}{l}0.036 \\
\text { II-IV }\end{array}$ \\
\hline BPDD & $78.0 \pm 7.8$ & $75.9 \pm 8.3$ & $68.0 \pm 8.5$ & $78.4 \pm 11.0$ & NS \\
\hline HRD & $83.6 \pm 9.6$ & $81.4 \pm 8.8$ & $74 \pm 8.6$ & $85.5 \pm 10.4$ & NS \\
\hline BPSN & $103.9 \pm 15.5$ & $108.1 \pm 18.9$ & $117.0 \pm 15.6$ & $128.7 \pm 30.4$ & $\begin{array}{l}0.033 \\
\text { II-IV }\end{array}$ \\
\hline BPDN & $64.7 \pm 6.6$ & $66.0 \pm 10.5$ & $58.0 \pm 116$ & $67.9 \pm 20.4$ & NS \\
\hline \multirow[t]{2}{*}{ HRN } & $73.2 \pm 7.0$ & $70.5 \pm 10.1$ & $62.0 \pm 8.0$ & $73.3 \pm 8.3$ & NS \\
\hline & \multicolumn{5}{|c|}{ Males } \\
\hline BPSD & $137.9 \pm 18.6$ & $134.7 \pm 12.5$ & $133.8 \pm 8.5$ & $140.1 \pm 15.7$ & NS \\
\hline BPDD & $82.7 \pm 11.9$ & $81.2 \pm 8.9$ & $79.2 \pm 8.0$ & $80.5 \pm 10.2$ & NS \\
\hline HRD & $84.2 \pm 8.1$ & $84.2 \pm 13.8$ & $84.2 \pm 8.5$ & $83.6 \pm 9.6$ & NS \\
\hline BPSN & $124.7 \pm 24.1$ & $119.3 \pm 22.7$ & $123.0 \pm 16.9$ & $127.5 \pm 16.5$ & NS \\
\hline BPDN & $68.7 \pm 9.8$ & $70.0 \pm 10.6$ & $69.4 \pm 11.8$ & $67.5 \pm 10.9$ & NS \\
\hline HRN & $67.4 \pm 6.9$ & $67.4 \pm 10.6$ & $67.6 \pm 8.3$ & $73.2 \pm 7.0$ & NS \\
\hline
\end{tabular}

$\mathrm{BP}$ - blood pressure;

BPDD - diastolic blood pressure during the day; BPSN - systolic blood pressure during the night; HRN - heart rate during the night;
BPSD - systolic blood pressure during the day;

HRD - heart rate during the day;

BPDN - diastolic blood pressure during the night;

NS - not significant. 
workers from group IV showed an increased systolic BP during the day, but the difference was insignificant. During the night, increased systolic BP was observed in six (21.4\%) workers from group I, eight (18.2\%) from group II, three $(37.5 \%)$ from group III, and 11 (47.8\%) workers from group IV. The between-group difference was statistically significant $(\mathrm{p}=0.015)$. The increased diastolic $\mathrm{BP}$ during the day and the night was recorded in a few workers, but the difference was statistically insignificant. The non-deeper phenomenon was detected in 31 (37\%) workers. Percentage of non-deepers in particular groups did not differ significantly.

The analysis of blood pressure by gender revealed that in the female workers, BPSD and BPSN were significantly higher in group IV than in group II. In male workers, these variables were also higher in group IV, but the difference was not significant (Table 4).

Before CPT, systolic BP in group I (148.4 \pm 19.0$)$ was found to be significantly higher than in groups II (131.8 \pm 15.6) and IV (132.4 \pm 16.4$)(p=0.0011)$. No differences were detected in diastolic BP or HR. After the test, systolic BP in group I was significantly higher than in group IV $(147.1 \pm 18.2$ vs. $134.9 \pm 14.4)(p=0.0433)$.

The CPT results revealed that 50 workers showed the increased and 44 ones the decreased BPS values. Most of the workers (66 vs. 28), presented the increased BPD after the cold pressor test.

The number of subjects responding to CPT by blood pressure growth was twice as high in female workers than in male workers (24 vs. 12). At the same time, more male workers (32 vs. 26) reacted to this test by a decreased BPS.

In the group of workers (18 males and 5 females) with elevated blood pressure observed in ABPM and/or earlier diagnosed, the male workers responded to CPT either by increased or decreased BP, while all female workers responded by the increased BP. The age of the male and female workers with hypertension did not differ significantly.

The analysis of time-domain HRV parameters did not show any statistically significant differences between the study groups. Slight, insignificant differences $(p=0.065)$ in R-R standard deviation were observed between groups III and
Table 5. Linear regression model

\begin{tabular}{lccl}
\hline HRV parameters & $\begin{array}{c}\text { Regression } \\
\text { coefficient }\end{array}$ & $\begin{array}{c}\text { Standard error } \\
\text { of coefficient }\end{array}$ & P \\
\hline BPS_B (VLF) & 1.53 & 0.47 & 0.016 \\
BPS_B (HF) & -0.53 & 0.28 & 0.06 \\
BPD_B (HF) & -0.38 & 0.15 & 0.013 \\
HR_B (HF) & -0.55 & 0.19 & 0.0054 \\
BPS_O (VLF) & 1.76 & 0.38 & 0.0002 \\
BPD_O (VLF) & 0.80 & 0.31 & 0.0107 \\
HR_O(VLF) & 0.74 & 0.24 & 0.0025 \\
BPD_O (HF) & -0.39 & 0.18 & 0.0332 \\
HR_O (HF) & -0.58 & 0.13 & 0.0000 \\
HR_O (LF/HF) & 7.47 & 2.06 & 0.0005 \\
\hline
\end{tabular}

BPS_B - systolic blood pressure before cold pressor test;

BPD_B - diastolic blood pressure before cold pressor test;

HR ${ }^{-}$- heart rate before cold pressor test;

BPS_O - systolic blood pressure (office measurement);

BPD_O - diastolic blood pressure (office measurement);

HR_O - heart rate (office measurement);

$\overline{\mathrm{HF}}$ - power spectrum within high frequency range $(0.15-0.35 \mathrm{~Hz})$;

VLF - power spectrum within very low frequency range, below $0.05 \mathrm{~Hz}$;

$\mathrm{LF} / \mathrm{HF}$ - sympathovagal balance ratio.

IV. However, group III embraced the oldest subjects, which could explain the lowered HRV in this group (STD R-R). The spectral analysis revealed that the power spectrum in all bands (VLF, LF, and HF) did not differ in the examined groups. Multiple linear regression analysis was used to assess how the neurovegetative regulation can affect the cardiovascular response to the work in cold environment. After CPT, the positive correlation between VLF and systolic BP (BPS_A) $(p=0.0076)$ and the negative one between HF and systolic BP (BPS_A) or heart rate (HR_A) ( $p=0.058$ and $p=0.0007$, respectively) were found. We also observed statistically significant correlations between HRV parameters, $\mathrm{HR}$, and $\mathrm{BP}$ before the cold pressor test and $\mathrm{BP}$ and $\mathrm{HR}$ measured on office examination (BPS_O, BPD_O, HR_O). The details of this analysis are shown in Table 5. Negative correlation of borderline significance $(p=0.06)$ was found between VLF and ambient temperature in workers exposed to temperature of $0-10^{\circ} \mathrm{C}$ at work (group IV).

\section{DISCUSSION}

Some authors report that occupational exposure to cold environment may contribute to the development of arterial hypertension [4]. However, our results showed that 
the prevalence of hypertension in workers occupationally exposed to cold microclimate was lower than in the adult population of Poland (25\% vs. 29\%). This can be attributed to the "healthy worker effect," i.e. the fact that people with hypertension avoid jobs associated with exposure to cold environment, whereas for healthy ones this does not make much difference.

Our analysis of CPT results indicated that female workers were more sensitive to cold-related stimuli, and those with hypertension were particularly sensitive; they responded to CPT by the increased BP. No relationship like that was detected in the male workers. The results of ABPM also showed that in male and female workers, the systemic response to work under conditions of cold microclimate was different. In the studies performed by Kasagi [22] in the general population of Japan, the BP response to CPT was more evident in men than in women.

It was also important whether the work was performed at temperatures below or above $0^{\circ} \mathrm{C}$. In the workers employed at very low temperatures, the systolic BP was similar before and after CPT, indicating their good adaptability to low-temperature conditions. On the other hand, those employed at temperatures of up to $14^{\circ} \mathrm{C}$ (groups II and IV) had a slightly higher systolic BP after the test, which may indicate their higher sensitivity to cold. The between-group comparison of ABPM values revealed that the physiological response was stronger for prolonged $(8 \mathrm{~h})$ exposure to moderately low temperatures $\left(0-10^{\circ} \mathrm{C}\right)$ than for short-term (about $30 \mathrm{~min}$ ) exposure to very low $\left(-26^{\circ} \mathrm{C}\right)$ temperatures. In the subjects working for $8 \mathrm{~h}$ at $0-10^{\circ} \mathrm{C}$, higher systolic BP values were noted not only in the daytime, but also at night. This would mean that the effects of stress associated with exposure to low-temperature prevail not only during the exposure period, but also afterwards. A higher BP at night as well as lack of drop of BP during night is an unfavorable prognostic symptom indicative of a higher risk of left ventricle hypertrophy and of other hypertension complications [23]. This symptom could be detected only by ABPM. The ABPM method may prove to be a useful tool in occupational medicine as it offers the possibility of monitoring changes in BP associated with work performance.

The lack of significant differences in HRV parameters between the groups exposed to different environmental conditions evidenced the workers' good tolerance for such working conditions. This was supported by the results of multiple regression analysis showing that $\mathrm{HRV}$ was modulated rather by age than by exposure parameters (duration of exposure to cold environment, workplace temperature, and energy expenditure).

The HRV analysis indicated that arterial BP and HR in people exposed to low ambient temperatures were modulated by the very low- and high-frequency components of the R-R spectrum. Information on the slowest (VLF, below $0.05 \mathrm{~Hz}$ ) component is scarcely available; it is supposed to be affected by parts of the autonomic nervous system as well as by the thermoregulatory processes and the renin/angiotensin system [5,6]. However, the latter relationship is disputable to some authors [12].

The renin/angiotensin system plays an important role in the pathogenesis of arterial hypertension through angiotensin II acting on blood vessels [24]. The negative correlation between HF power spectrum and systolic BP or HR in the study population after CPT indicated that the parasympathetic activity decreased in response to the stress evoked by low temperature. Our findings imply that the exposure-related BP variation in the study population could be associated with the increased activity of the renin/angiotensin system (VLF spectrum power) and with the decreased parasympathetic activity (HF).

The results of our earlier studies in workers exposed to electromagnetic fields provide evidence for a significant positive relationship between the level of arterial blood pressure and VLF power spectrum [25,26]. The present findings confirm our earlier observations. The negative correlation between arterial blood pressure at ABPM and HF power spectrum points to a significant role of the parasympathetic system in blood pressure regulation. Increased HF activity is related to both lower BP and lower HR. This supports a hypothesis on the protective role of the parasympathetic system in cardiovascular functions. The positive relationship found between the LF/HF ratio and HR confirms the conjecture that increased HR is associated with the dominant activity of the sympathetic nervous system in the neurovegetative regulation. The correlation between the activity of the autonomic nervous 
system within the VLF band and the arterial blood pressure after CPT indicates the significance of this frequency range in thermoregulatory processes.

Our findings cannot be compared with the results of other investigations since to our best knowledge no such studies have been carried out to date. However, single reports from experimental studies are available. For example, Fleisher et al. [27] demonstrated that the decreased temperature was associated with the increased VLF power spectrum

\section{CONCLUSIONS}

The results of our study indicated that the reaction of cardiovascular system in workers exposed to cold microclimate depended on gender and ambient temperature. Women seemed to be more sensitive to cold stress than men. However, owing to a small number of the subjects examined, this finding must be further investigated in a larger study. The lack of differences in HRV parameters between the study groups showed the workers' good tolerance for such working conditions. This might have been due also to the fact that the workers examined wore appropriate protective clothing, which was confirmed by the calculated IREQ value.

\section{REFERENCES}

1. Park H, Jung S, Moon D, Lee J, Kim J, Choi J, et al. Study about health hazards in cold exposed workers. Kor J Occup Med 1999;11:80-94.

2. Van Bergen P, Fregly MJ, Rossi F, Shechtman O. The effect of intermittent exposure to cold on the development of hypertension in the rat. Am J Hypertens 1992;5:548-55.

3. Kim H, Kang C, Han S, Kim S, Jo K. Effects of low ambient temperature to renal function and atrial diuretc hormones. Kor J Prev Med 1993; 8:134-40.

4. Kim JY, Jung KY, Hong YS, Kim JII, Jang TW, Kim JM. The Relationship between cold exposure and hypertension. J Occup Health 2003;45:300-6.

5. Bieniaszewski L. Physiological mechanisms of heart rate control. In: Piotrowicz R, editor. Heart Rate Variability. Gdańsk: Via Medica; 1995. p. 5-13 [in Polish].

6. Malik M. Heart rate variability, standards of measurement, physiological interpretation, and clinical use. Circulation 1996;5:1043-65.
7. Murata K, Landrigan PJ, Araki S. Effects of age, heart rate, gender, tobacco and alcohol ingestion on R-R intervals variability in human ECG. J Auton Nerv Syst 1992;37:199-206.

8. Hayano J, Yamada A, Mukari S, Sakakibara Y, Yamada M. Severity of coronary atherosclerosis correlates with the respiratory component of heart rate variability. Am Heart J 1991;121:1070-9.

9. Muhlnickel B. The value of heart rate frequency variability in the prognostic evaluation of patients with severe cerebral injuries. Anaesthesiol Reanim 1990;15:342-50.

10. Van Ravenswaaij-Arts CM, Kollee LA, Hopman JC, Stoelinga GB, Van Geijn HP. Heart rate variability. Ann Int Med 1993;118:436-47.

11. Singer DH, Martin GJ, Magid N, Weiss JS, Schaad JW, Kechoe R, et al. Low heart rate variability and sudden cardiac death. J Electrocardiol 1988;21(Suppl):46-55.

12. Trzebski A, Smietanowski M. Cardiovascular periodicities in healthy humans in the absence of breathing and under reduced chemical drive of respiration. J Auton Nerv Syst 1996;57:144-8.

13. Brenner IK, Thomas S, Shephard RJ. Autonomic regulation of the circulation during exercise and heat exposure. Inferences from heart rate variability. Sports Med 1998;26:85-99.

14. Kristal-Boneh E, Reifel M, Froom P, Ribak J. Heart rate variability in health and disease. Scand J Work Environ Health 1995;21:85-95.

15. Mancia G. Ambulatory blood pressure monitoring: research and clinical implication. J Hypertension 1990;8(Suppl 7):1-13.

16. Meyer-Sabellek W, Schulte K, Liederwald K, Van Gemmeren D, Gotzen R. Blood pressure profile and cardiac risk in hypertensive patients with left ventricular hypertrophy. J Hypertension 1990;8:595-8.

17. Pickering TG, O'Brien E. Consensus and Conclusions. The Second International Consensus Meeting on Twenty-Four-Hour Ambulatory Blood Pressure Measurment. J Hypertension 1991; (Suppl 8): 2-6.

18. Siegel WC, Blumanthal JA, Divine GW. Physiological, psychological and behavioral factors and white coat hypertension. J Hypertension 1990;16:140-6.

19. Pickering T, James G, Boddie C, Harshfield G, Blank S, Laragh JH. How common is white coat hypertension? JAMA 1988;259225-31.

20. Ohkubo T, Imai Y, Nagai K, Watanabe N, Minami N, Itoh O, et al. Prognostic significance for mortality among "white coat" and "reversed white coat" hypertension [abstract]. Ann Noninvas Electro 1996;1:4-13.

21. Staessen J, Fagard R, Lijnen P, Thijs L, Van Hoof R, Amery A. Reference values for ambulatory blood pressure: a meta-analysis. J Hypertension 1990;8(Suppl 6):57-64.

22. Kasagi F. Prognostic value of the cold pressor test for hypertension based on 28-year follow-up. Hiroshima J Sci 1994;43:93-103. 
23. Hoshide S, Kario K, Hoshide Y, Umeda Y, Hashimoto T, Kunii O, et al. Associations between nondipping of nocturnal blood pressure decrease and cardiovascular target organ damage in strictly selected community-dwelling normotensive. Am J Hypertens 2003;16:434-8.

24. Malliani A, Lombardi F, Pagani M. Power spectrum analysis of heart rate variability: a tool to explore neural regulatory mechanisms. $\mathrm{Br}$ Heart J 1994;71:1-2.
25. Bortkiewicz A, Zmyślony M, Gadzicka E, Pałczyński C. Ambulatory ECG monitoring in workers exposed to MF electromagnetic fields. J Med Eng Technol 1997;21:41-6.

26. Bortkiewicz A, Gadzicka E, Szymczak W. Heart rate variability in workers exposed to carbon disulfide. J Auton Nerv Syst 1997;66:62-8.

27. Fleisher LA, Frank SM, Sessler DI, Cheng C, Matsukawa T, Vannier CA. Thermoregulation and heart rate variability. Clin Sci (Lond) 1996;90:97-103. 